\title{
Transmisión de la malaria urbana en Buenaventura, Colombia: aspectos entomológicos ${ }^{1}$
}

\author{
Víctor Olano,2 Gabriel Carrasquilla ${ }^{3}$ y Fabián Méndez ${ }^{4}$
}

RESUMEN En los últimos años, el número de casos de malaria urbana en Buenaventura, Colombia, ha aumentado de 576 en 1987 a 3296 en 1991 y 2017 en 1992, por lo que se desarrolló un estudio epidemiológico para identificar patrones de transmisión de la malaria en este municipio del litoral pacífico colombiano. Se describen los hallazgos entomológicos en relación con los vectores, los criaderos y el comportamiento de las especies de Anopheles durante el período comprendido entre junio de 1993 y mayo de 1994. De los 469 posibles criaderos del área urbana, 28 fueron positivos a anofelinos. En la zona rural vecina de Buenaventura 20 de 80 posibles criaderos fueron positivos a las formas inmaduras del mosquito. Los sitios donde se encontró con más frecuencia Anopheles albimanus fueron las excavaciones de minería, los lagos y los estanques para piscicultura. Los criaderos de Anopheles nuñeztovari fueron los pozos de lluvia y estanques de peces. Anopheles neivai se recolectó en bromelias. De las capturas intra y peridomiciliarias de mosquitos adultos, 90\% correspondieron a Anopheles albimanus. De las hembras, 54,8\% habian realizado al menos una ovipostura. Esta especie tuvo una actividad peridomiciliaria máxima entre las 18.00 y 22.00 horas. La mayor tasa de picadura peridomiciliaria fue de 7,1 y correspondió a marzo de 1994. Se espera que esta información entomológica permita poner en marcha un importante proceso de participación comunitaria e implementar medidas de control que estén orientadas por nuestros resultados.

La malaria es una enfermedad tropical endémica responsable de uno de los principales problemas de salud pública de Colombia. Entre los factores que mantienen el carácter endémico de esta enfermedad en vastas zonas del país figuran la resistencia del pará-

\footnotetext{
1 Próximamente se publicará una versión en inglés de este artículo.

2 Instituto Nacional de Salud, Santa Fe de Bogotá, Colombia.

3 Universidad del Valle, Departamento de Microbiología, Cali, Colombia. Toda correspondencia debe dirigirse a este autor a la siguiente dirección postal: Carrera 5a. \#6-05, Cali, Colombia. Fax: 57-2883-4706.

4 Secretaría Departamental de Salud del Valle, Cali, Colombia.
}

sito a las drogas antimaláricas; la migración de poblaciones no inmunes a áreas palúdicas; las condiciones socioeconómicas y culturales de los colonos, como el tipo de vivienda; la resistencia a las campañas antimaláricas; las políticas, estrategias y recursos para el control de la enfermedad; el orden público, y la resistencia del vector a los insecticidas (1). Sin embargo, uno de los factores que más ha contribuido a mantener esta enfermedad en nuestro medio es que se desconoce el comportamiento biológico de las diferentes especies de Anopheles. Este conocimiento es necesario para determinar la dinámica de transmisión de esta enfermedad en cada zona en particular y así poder implementar medidas de control contra los anofelinos. En el país se han realizado algunos estudios sobre estos aspectos (2-6), pero es necesario hacer más investigaciones entomológicas y epidemiológicas sobre esta enfermedad.

Según el Servicio de Erradicación de la Malaria (SEM), en Colombia están registradas 41 especies de Anopheles. ${ }^{5}$ De esta rica fauna de anofelinos, aquí

\footnotetext{
5 Suárez MF, Quiñones MS, Fleming GA. Revisión de algunos aspectos entomológicos de malaria en Colombia. Documento interno del Servicio de Erradicación de la Malaria; 1988.
} 
se examinan los tres vectores principales - Anopheles (Nyssorhynchus) albimanus, Anopheles (Nyssorhynchus) darlingi y Anopheles (Nyssorhynchus) nuñeztovari- y los cuatro vectores secundarios: Anopheles (Anopheles) punctimacula, Anopheles (Nyssorhynchus) pseudopunctipennis, Anopheles (Kerteszia) lepidotus y Anopheles (Kerteszia) neivai (2).

En el litoral del Pacífico se han identificado 21 especies de Anopheles (2, 7-9), de las cuales se han registrado en la cabecera municipal de Buenaventura las especies $A$. $(N)$ albimanus, $A$. (N) punctimacula y $A$. (K) neivai ${ }^{6}$ (10). De ellas, $A .(N)$ albimanus se considera el principal vector de dicha costa $(3,11$, 12). Otra especie transmisora de la malaria en este territorio es $A$. (N) nuñeztovari $(4,9,13)$. Por otra parte, un informe de Cristóbal Viveros (SEM, documento interno, marzo de 1993) sobre una evaluación entomológica llevada a cabo en 26 localidades del municipio de Buenaventura en el período de 1987 a 1991 indica que las especies A. albimanus, $A$. neivai y $A$. nuñeztovari son las que mantienen activa la transmisión de la malaria en estos lugares.

En la ciudad de Buenaventura, principal puerto colombiano sobre la costa del Pacífico, se venía registrando un gran número de casos de malaria (576 en 1987, 3296 en 1991 y 2017 en 1992) (14). Por esta razón se inició un proyecto en el que se aplicó la estrategia de atención primaria para el control de la malaria en el área urbana de Buenaventura. La estrategia abarcó el diagnóstico de casos y la atención integral, teniendo en cuenta los aspectos entomológicos y epidemiológicos del problema, las características de los servicios de salud y los conocimientos, creencias y prácticas de la población en relación con la malaria. El estudio entomológico se adelantó con el propósito de identificar las especies de Anopheles que servirían de vectores de la malaria en el área urbana de Buenaventura, así como sus hábitos, tasa de picadura y tipos de criaderos.

\footnotetext{
Muñoz F. Anopheles (K) neivai como vector de la malaria en el municipio de Buenaventura. [Tesis en Ciencias de la Educación]. Bogotá: Escuela Normal Superior de Bogotá; 1974.
}

\section{MATERIALES Y MÉTODOS}

La ciudad de Buenaventura, que se encuentra a $7 \mathrm{~m}$ sobre el nivel del mar, tiene una temperatura promedio de $28^{\circ} \mathrm{C}$ y una precipitación media anual de $7789 \mathrm{~mm}$. Su área municipal es de $6078 \mathrm{~km}^{2}$ y se sitúa a $142 \mathrm{~km}$ de la ciudad de Cali (15). Este estudio se realizó en el área urbana de Buenaventura y en el área rural cercana (localidades de Sitronella, Zacarías, Gamboa, La Jaci, La Gloria y Variante Madroñal) entre junio de 1993 y mayo de 1994.

Se realizó un barrido completo del área urbana del municipio de Buenaventura y de la zona rural cercana para identificar todos los posibles criaderos naturales y artificiales de Anopheles. Se utilizó la metodología recomendada por la OMS (16), que consiste en sumergir 10 veces un cucharón de $250 \mathrm{~cm}^{3}$ por cada metro cuadrado de superficie de la colección de agua que se está investigando. Las larvas recolectadas y rotuladas se guardaron en viales de vidrio con alcohol al 70\%. Se llevaron a cabo dos ciclos de inspecciones larvales en el área urbana: el primero en junio y julio de 1993 y el segundo en diciembre de 1993 y enero de 1994. En el área rural estas inspecciones se realizaron en agosto y septiembre de 1993 en las seis localidades ya mencionadas y en diciembre de 1993 y enero de 1994 solo se realizaron en las localidades en que se habían encontrado criaderos. Todos los registros entomológicos se consignaron en formularios diseñados para esta investigación. El material recolectado se procesó en el laboratorio según la metodología de Forattini (17) y se identificó por medio de las claves de Suárez et al. (18) y Cova García (19).

La captura de anofelinos con cebos humanos es el método que con más frecuencia se usa para el muestreo de poblaciones de mosquitos, ya que permite evaluar el grado de contacto entre los seres humanos y el vector y los hábitos de picadura, densidad, tasa de esporozoitos y tasa de paridad de los mosquitos (20). La metodología utilizada consistió en seleccionar lugares de muestreo en los diferentes barrios del área urbana y en el área rural cercana, de acuerdo con el número de ca- sos de malaria registrados y de la presencia de criaderos cercanos al lugar escogido para la captura. Hubo dos recolectores, uno en el exterior y otro en el interior de las casas. Se utilizó la metodología recomendada por la OMS para la captura de anofelinos con cebos humanos (16). Los mosquitos se colocaron en recipientes plásticos divididos en lotes de acuerdo con la hora en que fueron recolectados, se empacaron, rotularon y se llevaron al laboratorio para su identificación. Las capturas se llevaron a cabo tres veces por semana entre las 18.00 y 24.00 horas. Durante octubre y noviembre la recolección de mosquitos se realizó entre las 18.00 y las 6.00 horas.

Se utilizó la trampa de Shannon (21) a base de luz blanca y un recolector que actúa como cebo, entre las 18.00 y 22.00 horas durante 8 semanas. La trampa de luz CDC (de los Centros para el Control y la Prevención de las Enfermedades, Atlanta, Georgia, Estados Unidos de América) (22) se colocó durante toda la noche (de las 18.00 a las 6.00 horas) para obtener muestras de las especies de anofelinos presentes en el área. Esta trampa se colocó dentro y fuera de los domicilios y en la proximidad de los criaderos.

Se probó la trampa de Mitchell modificada (23), colocándose en ella un ternero durante toda la noche (de las 18.00 a las 6.00 horas). Al día siguiente los mosquitos que quedaron atrapados se recolectaron, se empacaron y se transportaron al laboratorio para su identificación.

Con objeto de determinar los hábitos de reposo de los anofelinos (21), se hizo una búsqueda de estos mosquitos de las 6.00 a las 7.00 horas en el interior de las casas. Para identificar sus lugares de reposo naturales se inspeccionó la vegetación durante el día.

También se realizaron recolecciones en corrales (capturas en abrigo animal) (21) entre las 18.00 y las 22.00 horas debido a la importancia que tiene este método de captura para los estudios de densidad de anofelinos, en particular de A. albimanus. Aunque Buenaventura no es una zona ganadera, se localizó un establo en el barrio La Unión de la comuna 12, relativamente 
cerca del lugar donde se recolectaron los mosquitos adultos con cebos humanos. De julio de 1993 a abril de 1994 se recolectaron una vez a la semana los anofelinos que picaban al ganado o que reposaban sobre las paredes del establo.

En el laboratorio, el material recolectado e identificado se procesó con la técnica de inmunoensayo enzimático (ELISA) para determinar su infectividad con Plasmodium. Se usó, además, la técnica de Detinova (24) para disecar los ovarios de A. albimanus recolectados con cebos humanos y así determinar su edad fisiológica.

Se utilizaron métodos estadísticos descriptivos para las especies más frecuentes y la distribución de criaderos. También se elaboraron cuadros y gráficos mediante la utilización del programa Harvard Graphics (25). Para determinar la relación entre la densidad de los mosquitos en períodos de precipitación pluvial y la frecuencia de casos de malaria en la zona urbana se utilizaron el coeficiente de correlación de Pearson (26) y el programa de computación Stata 3.1 (27).

\section{RESULTADOS}

\section{Localización de criaderos e identificación de formas inmaduras}

En el área urbana de Buenaventura se identificaron 469 posibles criaderos de Anopheles (cuadro 1), en 28 (6\%) de los cuales se encontraron formas inmaduras de estos mosquitos. En la comuna 12 se encontraron $26(93 \%)$ de los criaderos positivos y también el mayor número de casos de malaria. En el área rural (Sitronella, Zacarías y Gamboa) se detectaron 20 criaderos positivos de un total de 80 sitios sospechosos (figura 1). Las excavaciones para minería, los estanques para piscicultura y las lagunas fueron los criaderos donde con más frecuencia se hallaron larvas de $A$. albimanus y $A$. nuñeztovari. Se encontraron larvas de A. $(K)$ neivai en una de 27 bromelias examinadas. En el cuadro 2 se presenta la distribución de especies de 1483 larvas de Anopheles recolectadas en el área urbana y en el área rural adyacente.

\section{Muestreo de poblaciones de mosquitos adultos}

Durante esta investigación se realizaron dos inspecciones en busca de criaderos en el área urbana de Buenaventura. En la primera (junio de 1993) se encontraron 17 criaderos con mosquitos y en la segunda (enero de 1994) 11. Solamente seis de los criaderos que tuvieron larvas en el primer ciclo de inspección las tuvieron en el segundo muestreo, mientras que en el área rural cercana los 11 criaderos que fueron positivos en el primer ciclo lo siguieron siendo en el segundo. En el segundo muestreo de la zona rural circundante se registraron nueve criaderos nuevos.

Capturas con cebos humanos. Se recolectaron ocho especies de Anopheles mediante capturas con cebos humanos (cuadro 2). A. albimanus fue la especie más comúnmente encontrada en el área urbana y A. nuñeztovari la más frecuente en la zona rural, y lo mismo se observó en cuanto a la distribución de las formas inmaduras.

Al examinarse la densidad mensual de mosquitos adultos, $A$. albimanus presentó una tasa de picadura que varió entre 0 en agosto de 1993 y 7,1 en marzo de 1994 (figura 2), mientras que las demás especies presentaron tasas menores de 0,6. Las tasas más altas de picadura que tuvo $A$. albimanus ocurrieron dentro del domicilio en octubre de $1993(2,5)$ y marzo de $1994(7,1)$. A. nuñeztovari también es exofágico y su tasa más alta $(2,7)$ se encontró en Sitronella (figura 2). La figura 3, en que se comparan las tasas de picadura de A. albimanus observadas en el interior y exterior de las casas, muestra una mayor actividad en el exterior del domicilio. En ambos casos se presenta, además, una mayor actividad de picadura en las primeras horas de la noche.

La figura 4 muestra la relación entre la densidad de los mosquitos (tasa de picadura mensual durante un año) y la precipitación pluvial en Buenaventura

CUADRO 1. Criaderos inspeccionados y positivos a Anopheles en la zona urbana de Buenaventura, Colombia, y las zonas rurales cercanas, 1993 a 1994

\begin{tabular}{|c|c|c|c|c|c|c|c|c|c|c|}
\hline \multirow[b]{3}{*}{ Tipo de criadero } & \multicolumn{5}{|c|}{ Zona urbana } & \multicolumn{5}{|c|}{ Zona rural ${ }^{a}$} \\
\hline & \multicolumn{5}{|c|}{ No. de criaderos } & \multicolumn{5}{|c|}{ No. de criaderos } \\
\hline & Total & Positivos & $\begin{array}{l}\text { Anopheles } \\
\text { albimanus }\end{array}$ & $\begin{array}{l}\text { Anopheles } \\
\text { nuñeztovari }\end{array}$ & $\begin{array}{c}\text { Especies } \\
\text { combinadas }^{b}\end{array}$ & Total & Positivos & $\begin{array}{l}\text { Anopheles } \\
\text { albimanus }\end{array}$ & $\begin{array}{l}\text { Anopheles } \\
\text { nuñeztovari }\end{array}$ & $\begin{array}{c}\text { Especies } \\
\text { combinadas }^{b}\end{array}$ \\
\hline Excavaciones & 243 & 16 & 14 & 12 & 10 & 12 & 3 & $\ldots$ & 3 & $\ldots$ \\
\hline Estanques de piscicultura & 5 & 3 & 3 & 2 & 2 & 11 & 8 & 4 & 5 & 1 \\
\hline Quebradas y acequias & 35 & 2 & 2 & $\ldots$ & $\ldots$ & 10 & 1 & $\ldots$ & 1 & $\ldots$ \\
\hline Lagunas & 3 & 3 & 3 & 2 & 2 & 5 & 4 & 1 & 4 & 1 \\
\hline Bromelias & 27 & $1^{c}$ & $\ldots$ & $\ldots$ & $\ldots$ & $\ldots$ & $\ldots$ & $\ldots$ & $\ldots$ & $\ldots$ \\
\hline Otros (charcas, llantas, etc.) & 156 & 3 & 3 & $\cdots$ & $\cdots$ & 42 & 4 & & 4 & $\cdots$ \\
\hline Total & 469 & 28 & 25 & 16 & 14 & 80 & 20 & 5 & 17 & 2 \\
\hline
\end{tabular}


FIGURA 1. Distribución de criaderos positivos a Anopheles en el área urbana y en el área rural cercana de Buenaventura, Colombia, de 1993 a 1994

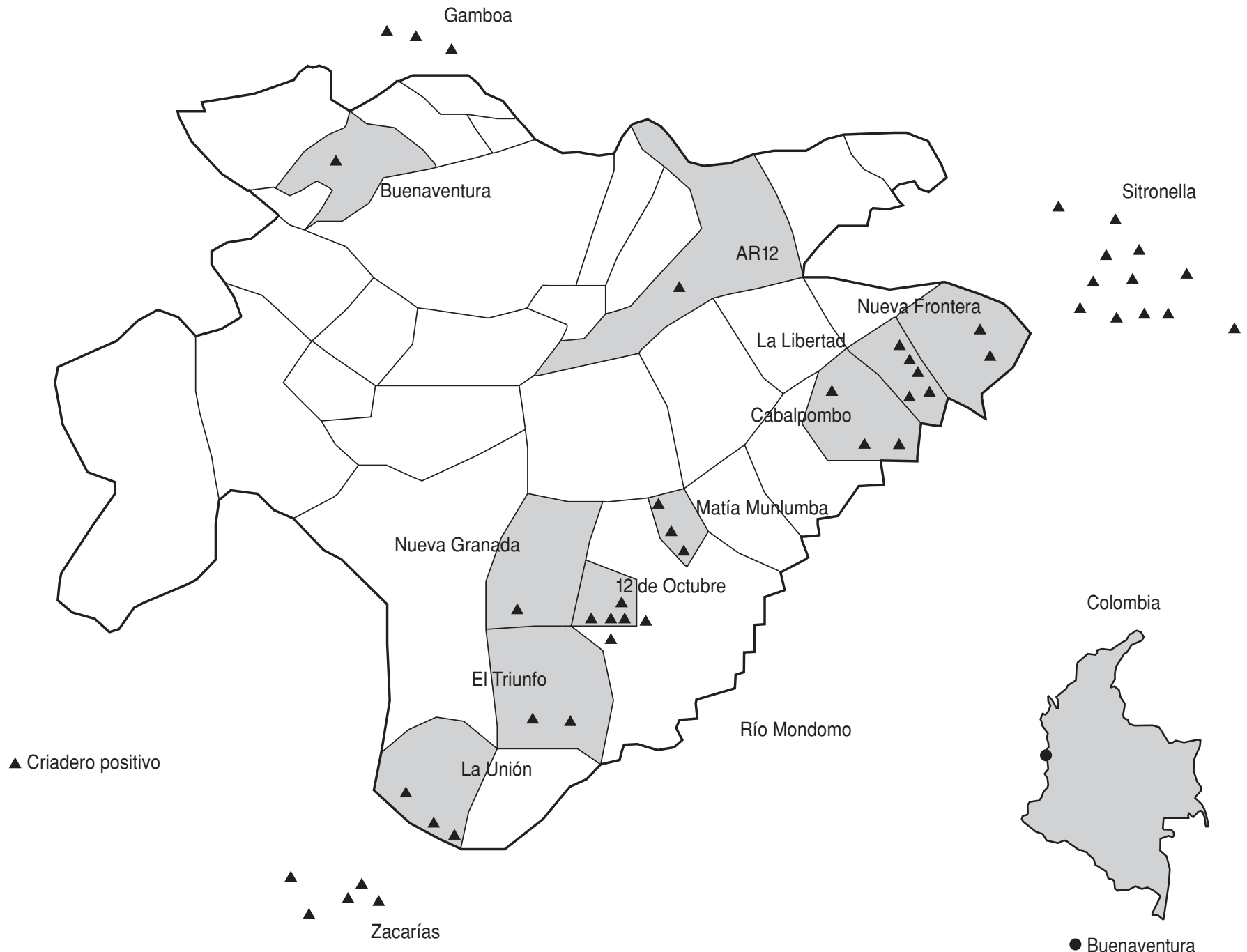

notificada por el Instituto Colombiano de Hidrología, Meteorología y Adecuación de Tierras (comunicación personal, agosto, 1994). La correlación entre la tasa de picadura y la precipitación pluvial durante el mes anterior no fue estadísticamente significativa cuando se analizó para todo el año $(r=$ $-0,35$; IC95\%: $-0,80$ a 0,36). Sin embargo, al examinarse solo hasta diciembre de 1993, se encontró un coeficiente de correlación de 0,87 (IC95\%: 0,21 a $0,99)$. De enero a mayo de 1994 el coeficiente es negativo $(-0,68)$, aun cuando no fue estadísticamente significativo (IC95\%: $-0,98$ a 0,51). Como indica la figura 4 , las tasas máximas de picadura que se observaron en octubre de 1993 y marzo de 1994 se presentaron uno y 3 meses después de los dos períodos de mayor precipitación pluvial.

Capturas con trampas. Durante el muestreo de las especies de Anopheles presentes en Buenaventura, que se llevó a cabo en junio y julio de 1993 con trampas de luz, no se capturaron mosquitos adultos. En cambio, con la trampa de Shannon se recolectaron las especies $A$. albimanus (nueve ejemplares) y $A$. neivai (dos ejemplares) durante junio, julio y agosto de 1993. Solamente se capturaron cinco ejem- plares de $A$. neivai con la trampa de Mitchell durante noviembre y diciembre de 1993.

Capturas de mosquitos en reposo en los interiores y en abrigo natural y establos. Durante las capturas de 36 horas en octubre de 1993 no se recolectaron mosquitos ni en los interiores ni en los corrales. En cuanto a mosquitos en abrigo animal, se capturaron 137 ejemplares de la especie $A$. albimanus, dos de $A$. neivai y uno de $A$. nuñeztovari. El mayor número de ejemplares de $A$. albimanus recolectado en una noche fue de 29 hembras. 
CUADRO 2. Número y porcentaje de formas inmaduras y adultas de Anopheles en la zona urbana de Buenaventura, Colombia y en la zona rural cercana, 1993 a 1994

\begin{tabular}{|c|c|c|c|c|c|c|c|c|c|}
\hline \multirow[b]{2}{*}{ Especie } & & & \multicolumn{6}{|c|}{ Zona rural } & \multirow[b]{2}{*}{ Total } \\
\hline & No. & $\%$ & No. & $\%$ & No. & $\%$ & No. & $\%$ & \\
\hline \multicolumn{10}{|l|}{ Formas inmaduras } \\
\hline A. albimanus & 453 & 75,6 & 4 & 0,7 & 80 & 55,9 & 200 & 100,0 & 737 \\
\hline A. nuñeztovari & 140 & 23,4 & 532 & 98,4 & 63 & 44,1 & 0 & - & 735 \\
\hline A. neivai & 2 & 0,3 & 0 & - & 0 & - & 0 & - & 2 \\
\hline Total & 599 & 100 & 541 & 100 & 143 & 100 & 200 & 100 & 1483 \\
\hline \multicolumn{10}{|l|}{ Formas adultas } \\
\hline A. albimanus & 2066 & 90,0 & 36 & 5,2 & 42 & 19,3 & 80 & 89,9 & 2224 \\
\hline A. nuñeztovari & 56 & 2,4 & 547 & 79,0 & 23 & 10,6 & 0 & - & 626 \\
\hline A. neivai & 132 & 5,7 & 48 & 6,9 & 116 & 53,2 & 6 & 6,7 & 302 \\
\hline A. rangeli & 12 & 0,5 & 20 & 2,9 & 19 & 8,7 & 0 & - & 51 \\
\hline Anopheles spp. & 17 & 0,7 & 32 & 4,6 & 12 & 5,5 & 3 & 3,4 & 64 \\
\hline Total & 2296 & 100 & 693 & 100 & 218 & 100 & 89 & 100 & 3296 \\
\hline
\end{tabular}

En Gamboa, otra vereda de la zona rural cercana, se encontró que $90 \%$ de los 80 mosquitos recolectados correspondían a $A$. albimanus, como en la zona urbana. Esto se explica porque esta vereda podría clasificarse como un barrio de Buenaventura. En Zacarías, en cambio, se encontraron $A$. $(K)$ neivai y A. albimanus (54\% y $20 \%$, respectivamente). La alta frecuencia de A. (K) neivai, especie de hábitos selváticos (13), se debe a la abundancia de bromelias, ya que esta localidad es la que se encuentra más distante del área urbana de Buenaventura y posee características más rurales. En Zacarías

FIGURA 2. Actividad de picadura de Anopheles albimanus y Anopheles nuñeztovari en el interior y exterior de los domicilios. Buenaventura, Colombia, de 1993 a 1994

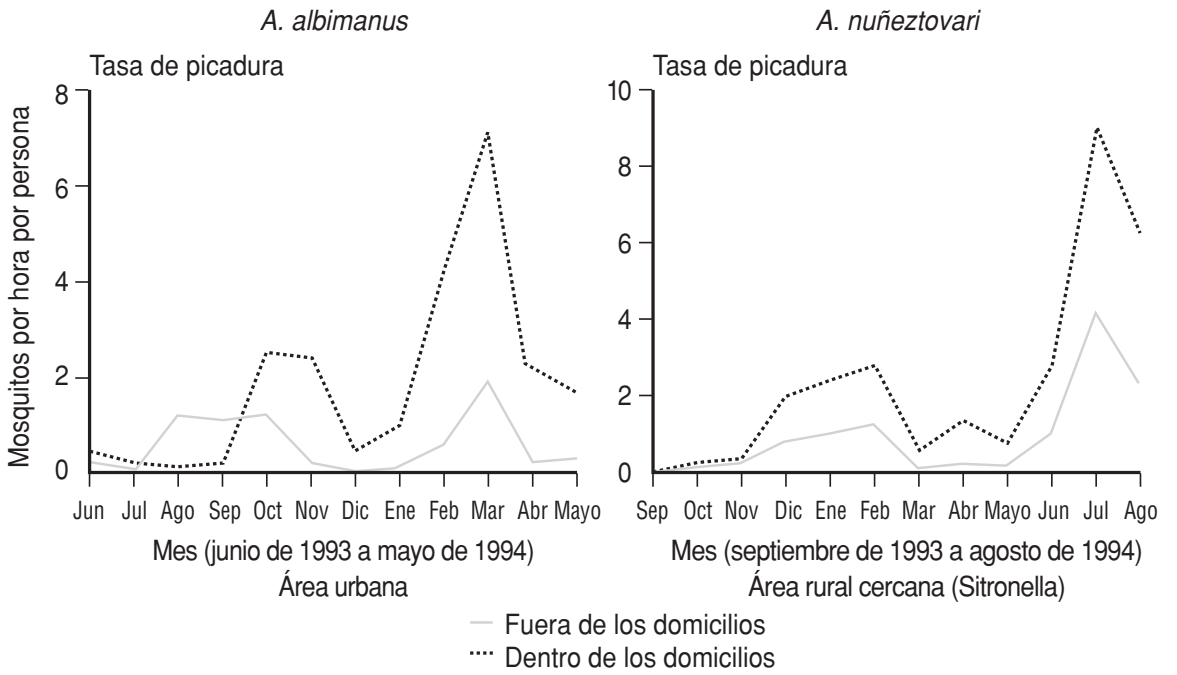

Casos de malaria y tasas de picadura. Durante el período de observación (figura 5) se encontró una asociación inversa $(r=-0,36$; IC95\%: $-0,79$ a 0,31$)$ pero no estadísticamente significativa, entre los casos de malaria durante un mes determinado y la tasa de picadura del mes anterior.

\section{DISCUSIÓN}

En el área urbana de Buenaventura se encontró $A$. albimanus en una gran variedad de criaderos, entre ellos excavaciones, estanques para peces, lagunas y charcos de agua pluvial, como 
FIGURA 3. Tasa de picadura de Anopheles albimanus de las 18.00 a las 24.00 horas, de junio de 1993 a mayo de 1994 en el área urbana de Buenaventura, Colombia

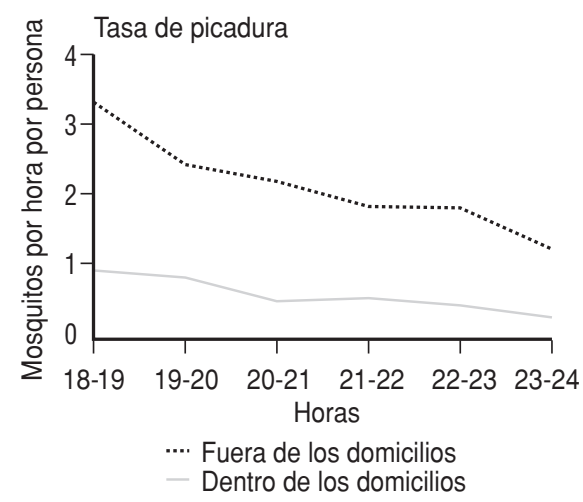

han observado Quiñones et al. (5) en otros lugares del litoral del Pacífico. Un hallazgo importante fue la presencia de $A$. (K) neivai en bromelias, donde suele habitar esta especie. Otros autores (4) han notificado resultados similares en el municipio de Charambira, Chocó, Colombia, donde se ha identificado $A$. $(K)$ neivai en bromelias y se ha incriminado a esta especie como vector de la malaria en esta área.

Se encontraron posibles criaderos en seis localidades rurales, pero solo en Sitronella, Zacarías y Gamboa se detectaron mosquitos. Los charcos con agua pluvial y los estanques para piscicultura fueron los lugares donde se identificaron con más frecuencia larvas de $A$. nuñeztovari y estas lagunas fueron los sitios que presentaron la mayor densidad de larvas por área de superficie. Esto coincide con lo detectado en otras áreas con malaria (28), donde también se han encontrado criaderos en estanques para el cultivo de peces con fines comerciales, resultado que deben tomar en cuenta las autoridades de salud pública al diseñar medidas de control.

La elevada persistencia de criaderos de mosquitos detectada en las inspecciones indica que se debe establecer dentro del programa de control un sistema permanente de vigilancia entomológica para detectar la aparición de
FIGURA 4. Fluctuaciones de la densidad de Anopheles albimanus y volumen de precipitación pluvial de junio de 1993 a mayo de 1994, en el área urbana de Buenaventura, Colombia

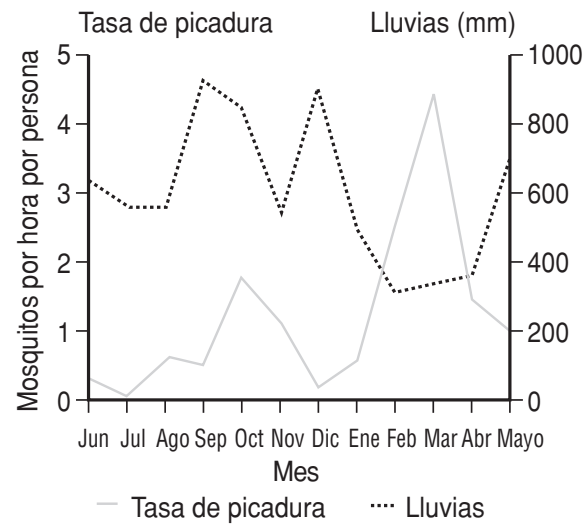

nuevos sitios de cría de mosquitos. Además, es necesario planear intervenciones con la participación activa de la comunidad, teniendo en cuenta las características de los criaderos.

$\mathrm{Al}$ igual que otros investigadores citados por Frederickson (20), encontramos que la tasa de picadura de $A$. albimanus aumentó después de los picos de precipitación pluvial. En Buenaventura esta especie mostró una tendencia exofágica, lo cual coincide con lo publicado en otros estudios $(5,29)$. Durante agosto y septiembre se registró una mayor actividad en el medio intradomiciliario, debido probablemente a la intensa lluvia que cayó durante algunos días de captura en agosto y a la realización incompleta de las actividades de recolección nocturna programadas en septiembre. El pico de actividad en el exterior de las viviendas se produjo entre las $18.00 \mathrm{y}$ las 21.00 horas y fue descendiendo hacia la medianoche, como se ha encontrado en El Salvador (30). Otros investigadores también han hallado picos de actividad antes de la medianoche en Centroamérica y el Caribe (20). A diferencia de lo hallado en este estudio, en Colombia (5) se ha notificado una actividad de picadura variable entre las 18.00 y 23.00 horas, tanto en el exterior como en el interior de las casas.
FIGURA 5. Fluctuaciones de la densidad de Anopheles albimanus y casos mensuales de malaria de junio de 1993 a mayo de 1994 en el área urbana de Buenaventura, Colombia

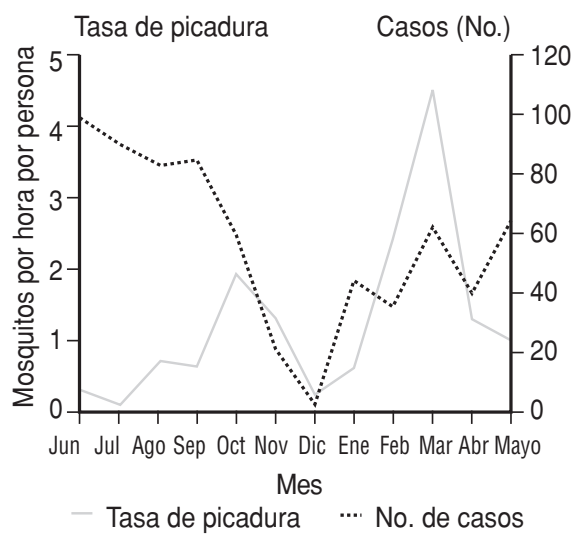

Durante octubre y noviembre de 1993 se realizaron capturas entre las 18.00 y las 6.00 horas en el medio intradomiciliario y entre las 18.00 y las 24.00 horas y las 5.00 y las 6.00 horas en el peridomiciliario, de acuerdo con la metodología sugerida por Elliot (31). En el interior de los domicilios se presentó un segundo pico de actividad entre las 24.00 y 1.00 horas, como han notificado otros investigadores (20). Entre las 5.00 y las 6.00 horas nuevamente se observó una mayor actividad peridomiciliaria que intradomiciliaria; asimismo, en Nicaragua, Rivera ${ }^{7}$ encontró que este mosquito presenta un segundo pico de actividad durante ese período. Sobre la base de los resultados obtenidos se puede concluir que $A$. albimanus tiene un comportamiento principalmente exofágico que es más acentuado en las primeras horas de la noche, lo cual también debe tenerse en cuenta cuando se realizan actividades de prevención de la malaria. Nuestros resultados en lo respectivo a la edad fisiológica de $A$. albimanus muestran una población muy joven sometida a la presión de rociamientos con insecticidas.

\footnotetext{
Rivera Mendoza P, López MM. Ecology and biology of Anopheles albimanus in a locality of the Pacific Coast of Nicaragua [abstr]. J Am Mosq Control Assoc 1991;7:635.
} 
En Sitronella, 79\% de los mosquitos adultos recolectados correspondieron a la especie A. nuñeztovari. Este mosquito, que en Colombia se considera el principal vector de la malaria, puede presentar dos patrones de picadura. El primero es típico de una especie exofágica y zoófila que pica al atardecer, mientras que el segundo es propio de especies antropófilas intradomiciliarias que pican tarde en la noche. Este último patrón se ha encontrado en el norte de Colombia y en el oeste de Venezuela (32) y es el que se asocia con el vector de la malaria humana. Tadei y Correia en Manaus, Brasil (33), encontraron que la especie $A$. nuñeztovari tenía hábitos exofílicos y antropófilos y que la actividad de picadura de esta especie llegaba a un máximo al atardecer, principalmente entre las 18.00 y 19.00 horas. En Suriname (34) se han encontrado resultados similares. En Buenaventura nuestros resultados concordaron con los de Tadei y Correia en el Brasil (33), donde la especie mostró hábitos de picadura predominantemente peridomiciliarios $\mathrm{y}$ una mayor actividad en las primeras horas de la noche.

Las capturas en abrigo animal, es decir, en corrales o establos, mostraron poca densidad de $A$. albimanus. La situación difiere de las otras zonas donde en un par de horas (18.00 a 20.00 horas) pueden recolectarse más de 1000 ejemplares de esta especie (V. Olano, datos inéditos). La carencia de ganado vacuno en esta zona hace, a su vez, que esta especie tenga hábitos antropófilos, y es posible que ello influya en la transmisión de la malaria en Buenaventura. Además, el no haber encontrado adultos en reposo natural confirma la dificultad de utilizar esta técnica como método de muestreo. Otros investigadores han obtenido los mismos resultados (20).

No encontramos una correlación estadísticamente significativa entre la tasa de picadura durante un mes determinado y el número de casos de malaria en el área urbana de Buenaventura. Otros investigadores (35) han señalado que el aumento del número de mosquitos después del período de lluvias disminuye la proporción de anofelinos infectados, lo cual implica que se reduce la transmisión de la malaria.

En este estudio se obtuvo información sobre los hábitos hematófagos, la tasa de picadura y el comportamiento del vector, que ha permitido diseñar una intervención para el control de la malaria en Buenaventura, Colombia, teniendo también en cuenta los conocimientos, actitudes, creencias y prácti- cas de la población y los factores de riesgo asociados con la malaria. Las actividades de vigilancia entomológica y el control integrado de los vectores comprenden no solo la destrucción de criaderos, sino el control físico y biológico del vector, la difusión de información y la educación de la comunidad.

Agradecimientos. Agradecemos al Programa Especial de Investigaciones y Enseñanzas sobre Enfermedades Tropicales de la OMS, y a la Fundación para la Educación Superior (Fundación FES) de Cali, Colombia, la financiación de este proyecto. Asimismo agradecemos al Instituto Nacional de Salud de Colombia; a la Unidad Administrativa Especial de Campañas Directas, en particular a los funcionarios y técnicos de la sede de Buenaventura; a la Secretaría de Salud Departamental del Valle del Cauca, y al Instituto de Salud del Pacífico (INSALPA), el apoyo técnico y logístico que nos brindaron durante la realización de esta investigación. Finalmente los autores expresamos nuestra gratitud a la población y a las autoridades municipales de las comunas 9, 10, 11 y 12 de Buenaventura y de las veredas Zacarías, Sitronella y Gamboa, por su apoyo y colaboración.

\section{REFERENCIAS}

1. González G. Memorias Seminario Taller Malaria: descentalización y control. Medellín: Servicio Seccional de Salud de Antioquia; 1991.

2. Herrera $S$, Suárez MF, Sánchez GI. Uso de la técnica inmuno-radiométrica (IRMA) en Anopheles de Colombia para la identificación de esporozoitos de Plasmodium. Colomb Med 1987;18:2-6.

3. Quiñones ML, Suárez MF, Rodríguez A, Fleming G, Galvis LE. Comportamiento de Anopheles (Kerteszia) lepidotus Zavortink 1973 y su incriminación como posible vector de la malaria en el departamento del Tolima, Colombia. Biomedica 1984;4: 5-13.

4. Carvajal H, Herrera MA de, Quintero J, Alzate A, Herrera S. Anopheles neivai: a vector of malaria in the Pacific lowlands of Colombia. Trans R Soc Trop Med Hyg 1989;5: 609-612.

5. Quiñones ML, Suárez MF, Fleming GA. Distribución y bionomía de los anofelinos de la
Costa Pacífica de Colombia. Colomb Med 1987;18:19-23.

6. Olano VA, Carrillo MP, Espinal CA. Estudios de infectividad de la especie Anopheles albimanus Wiedemann 1820 (Díptera: Culicidae) cepa Cartagena con plasmodios humanos. Biomedica 1985;5:5-10.

7. Gast Galvis A. Biología y distribución geográfica de los anofelinos en Colombia. Rev Fac Med 1943;12:53-103.

8. Barreto P. Distribución de mosquitos Anopheles en el departamento del Valle del Cauca. Acta Med Valle 1971;2:45-48.

9. Fajardo P, Barreto P, Suárez MF. Anofelinos de Córdoba (Buenaventura). Colomb Med 1983;14:99-103.

10. Colombia, Ministerio de Salud, Servicio de Erradicación de la Malaria. Vol II: Plan de Erradicación de la Malaria. Bogotá: Ministerio de Salud; 1957.
11. Ferro C. Paludismo: problema de salud pública en Colombia. Rev Esc Nac Salud Publica (Medellín) 1974;1:41-45.

12. Suárez MF. Aspectos entomológicos del paludismo en Colombia. Bol Epidemiol Antioquia 1985;10:142-143.

13. Fajardo P, Alzate A. Anopheles nuñeztovari como vector de la malaria en el Bajo Calima, Buenaventura. Colomb Med 1987; 18:14-18.

14. Méndez F, Carrasquilla G. Epidemiología de la malaria en el área urbana de Buenaventura: análisis de la ocurrencia en el período 19871993. Colomb Med 1995;26:77-85.

15. Instituto Geográfico Agustín Codazzi. Vol I: Diccionario geográfico de Colombia. Bogotá: Editorial Andes; 1971.

16. World Health Organization. Vol I: Manual on practical entomology in Malaria. Geneva: WHO; 1975. 
17. Forattini OP. Vol I: Entomología médica. São Paulo: Editora da Universidade de São Paulo; 1962.

18. Suárez MF, Quiñones ML, Robayo MA. Clave para la determinación taxonómica de larvas y adultos hembras de los principales anofelinos de Colombia. Bogotá: Ministerio de Salud; 1988.

19. Cova García P. Notas sobre los anofelinos de Venezuela y su identificación. 2a ed. Caracas: Grafos; 1961.

20. Frederickson EC. Bionomía y control de Anopheles albimanus. Washington DC: Organización Panamericana de la Salud; 1993. (Cuaderno técnico 34).

21. Service MW. A critical review of procedures for sampling populations of adult mosquitos. Bull Entomol Res 1977;67:343-382.

22. Sudia WD, Chamberlain RW. Battery-operated light trap: an improved model. Mosq News 1962;22:126-129.

23. Mekuria Y, Tidwell MA, Williams DC, Mandeville JD. Bionomic studies of the Anopheles mosquitoes of Dajabon Dominican Republic. J Am Mosq Control Assoc 1990;6:651-657.

24. Deinova TS. Age grouping methods in Diptera of medical importance with special reference to some vectors of malaria. Geneva: World Health Organization; 1962. (Monograph series 47).

25. Software Publishing Corporation. [Programa de computadora] Harvard Graphics 3.0. Mountain View, California; 1992.

26. Pagano M, Gauvreau K. Principles of biostatistics. Belmont, California: Duxbury Press; 1993

27. Stata Corporation. Stata reference manual, Release 3.1. 6a ed. College Station, Texas: Stata Corporation; 1993.

28. López D, González R. Análisis de la dispersión y la abundancia de los estadios larvales de Anopheles nuñeztovari (gabaldón) en estanques piscícolas del municipio de Buenaventura. Colomb Bol Mus Entomol Univ Valle 1994;2:73-84.

29. Bown D, Rios J, del Angel Cabañas G, Guerrero J, Méndez J. Evaluation of chlorphoxim used against Anopheles albimanus on the south coast of Mexico: results of indoor chlorphoxim applications and assessment of the methodology employed. Bull Pan Am Health Organ 1984;18(4):379-388.

30. Rachou R, Lyons G, Moura-Lima M, Austin Kerr J. Synoptic epidemiological studies of malaria in El Salvador. Am J Trop Med Hyg 1965;14:2-62.

31. Elliot R. Studies on the man-vector contact in some malarious areas of Colombia. Bull World Health Organ 1968;38:239-253.

32. Fleming G. Biología y ecología de los vectores de la malaria en las Américas. Washington DC: Organización Panamericana de la Salud; 1986. (PNSP/85-72).

33. Tadei PW, Correia JM. Biología de anofelinos amazónicos: IV, Observações sobre a actividade de picar de Anopheles nuñeztovari (Diptera: Culicidae). Acta Amazon 1982;12:71-74.

34. Panday RS. Anopheles nuñeztovari and malaria transmission in Surinam. Mosq News 1977;37: 728-737.

35. Astaiza R, Murillo C, Fajardo P. Biología de Anopheles (Kerteszia) neivai HD\&K (Diptera: Culicidae) en la costa pacífica de Colombia. Rev Saude Publica 1988;22:101-108.

Manuscrito recibido el 21 de julio de 1995 y aceptado para publicación en version revisada el 20 de marzo de 1996.

ABSTRACT During the past several years, there has been an increase in the cases of urban malaria in Buenaventura, Colombia, from 576 in 1987 to 3296 in 1991 and 2017 in 1992; therefore, an epidemiological study to identify transmission patterns was carried out in this municipality on Colombia's Pacific coast. This paper describes the entomological findings regarding vectors, potential breeding sites and behavior of Anopheles species during the period from June 1993 to May 1994. Of the 469 potential breeding sites identified in the urban area, 28 were positive for anophelines, while in the neighboring rural zone, 20 out of 80 potential breeding sites were positive for the immature forms of Anopheles. Mining excavations, lakes and breeding ponds for fish or shrimp were the places where A. albimanus was more frequently found. For $A$. nuñeztovari, the breeding sites were rain puddles and ponds for breeding fish or shrimp. A. neivai was also identified in the urban area and larvae were collected from bromeliaceous plants. Both inside and outside the households mosquito collections were carried out, and $90 \%$ of the collected mosquitoes were identified as $A$. albimanus. Of the female $A$. albimanus $54.8 \%$ were parous. This species was found to have peak peridomiciliary activity between 6 p.m. and 10 p.m. The highest biting rate in the peridomiciliary area was 7.1 and was observed during March. It is expected that this entomological information will permit the launching of a strong community participation process and the implementation of control measures in accordance with the entomological findings related to malaria in the urban area of Buenaventura. 